\title{
TAIGA observatory: IACT fabrication and tests
}

Y. Sagan ${ }^{4,8}$, I. Astapov ${ }^{3}$, P. Bezyazeekov ${ }^{1}$, A. Borodin ${ }^{4}$, M. Brueckner ${ }^{5}$, N. Budnev ${ }^{1}$, A. Chiavassa ${ }^{6}$, A. Dyachok ${ }^{1}$, O. Fedorov ${ }^{1}$, A. Gafarov ${ }^{1}$, A. Garmash ${ }^{7}$, V. Grebenyuk $^{4,8}$, O. Gress ${ }^{1}$, T. Gress ${ }^{1}$, A. Grinyuk ${ }^{4}$, O. Grishin ${ }^{1}$, D. Horns ${ }^{9}$, A. Ivanova $^{1}$, N. Kalmykov ${ }^{10}$, Y. Kazarina ${ }^{1}$, V. Kindin ${ }^{3}$, P. Kirilenko ${ }^{7}$, S. Kiryuhin ${ }^{1}$, R. $K_{\text {Kokoulin }}^{3}$, K. Kompaniets ${ }^{3}$, E. Korosteleva ${ }^{10}$, V. Kozhin ${ }^{10}$, E. Kravchenko ${ }^{7,11}$, M. Kunnas $^{9}$, L. Kuzmichev ${ }^{10}, 1$, Yu. Lemeshev ${ }^{1}$, V. Lenok ${ }^{1}$, N. Lubsandorzhiev ${ }^{10}$, B. Lubsandorzhiev $^{10,12}$, R. Mirgazov ${ }^{1}$, R. Mirzoyan ${ }^{13,1}$, R. Monkhoev ${ }^{1}$, R. Nachtigall ${ }^{9}$, E. Osipova ${ }^{1}$, A. Pakhorukov ${ }^{1}$, A. Pan ${ }^{4,16}$, M. Panasyuk ${ }^{10}$, L. Pankov ${ }^{1}$, A. Petrukhin ${ }^{3}$, V. Poleschuk' ${ }^{1}$, E. Popescu ${ }^{14}$, E. Popova ${ }^{10}$, A. Porelli ${ }^{5}$, E. Postnikov ${ }^{10}$, V. Prosin ${ }^{10}$, V. Ptuskin $^{15}$, A. Pushnin ${ }^{1}$, E. Rjabov ${ }^{1}$, G. Rubtsov ${ }^{12}$, B. Sabirov ${ }^{4}$, V. Samoliga ${ }^{1}$, A. Sidorenkov ${ }^{12}$, A. Silaev ${ }^{10}$, A. Silaev (junior) ${ }^{10}$, A. Skurikhin ${ }^{10}$, V. Slunecka ${ }^{4}$, A. Sokolov $^{7,11}$, Y. Suvorkin ${ }^{1}$, L. Sveshnikova ${ }^{10}$, V. Tabolenko ${ }^{1}$, A. Tanaev ${ }^{1}$, B. Tarashansky $^{1}$, L. Tkachev ${ }^{4,8}$, M. Tluczykont ${ }^{9}$, R. Wischnewski ${ }^{4,5}$, I. Yashin ${ }^{3}$, A. Zagorodnikov $^{1}$, D. Zhurov ${ }^{1,2}$

${ }^{1}$ Institute of Applied Physics ISU, Irkutsk, Russia, ${ }^{2}$ Irkutsk National Research Technical University, Irkutsk, Russia, ${ }^{3}$ National Research Nuclear University MEPhI (Moscow Engineering Physics Institute), Moscow, Russia, ${ }^{4}$ JINR, Dubna, Russia, ${ }^{5}$ DESY, Zeuthen, Germany, ${ }^{6}$ Dipartimento di Fisica Generale Universiteta di Torino and INFN, Torino, Italy, ${ }^{7}$ Novosibirsk State University, NSU, Novosibirsk, Russia, ${ }^{8}$ Dubna State University, Dubna, Russia, ${ }^{9}$ Institute for Experimental Physics, University of Hamburg, Germany, ${ }^{10}$ Skobeltsyn Institute of Nuclear Physics MSU, Moscow, Russia, ${ }^{11}$ Budker Institute of Nuclear Physics SB RAS, Novosibirsk, Russia, ${ }^{12}$ Institute for Nuclear Research of RAN, Moscow, Russia, ${ }^{13}$ MaxPlanck-Institute for Physics, Munich, Germany, ${ }^{14}$ ISS, Bucharest, Romania, ${ }^{15}$ IZMIRAN, Moscow, Russia, ${ }^{16}$ The Institute of Nuclear Physics, Almaty, Kazakhstan

E-mail: yaroslav sagan@mail.ru

Imaging Atmospheric Cherenkov Telescopes (IACTs) are one of the key parts of the TAIGA observatory, which is designed to study gamma rays and charged cosmic rays in the energy range of $10^{13} \mathrm{eV}-10^{18} \mathrm{eV}$. The current prototype phase comprises three IACTs. The first IACT was fabricated at JINR, Dubna, and started its first commissioning season in the Tunka valley in 2017. The second one has been assembled there in fall 2018, the third structure one is ready for installation in autumn 2019.

This report presents the main TAIGA IACT characteristics and results from pointing performance studies in the last 2 seasons. We also describe R\&D activities started to fabricate focusing glass mirrors at JINR, and give results for the first prototypes measurements.

36th International Cosmic Ray Conference -ICRC2019-

July 24th - August 1st, 2019

Madison, WI, U.S.A

*Speaker. 


\section{Introduction}

IACTs are the primary instruments for gamma-ray astronomy in the $\mathrm{TeV}$ energy range and are used in the ground-based experiments for Extensive Air Shower (EAS) measurements. IACTs allow the precise determination of the gamma-ray spectra using the EAS image analysis. In case of stereoscopic systems of multiple IACTs which observe EAS from different viewing angles, the EAS arriving angles can be obtained with a good precision by a simple geometrical reconstruction.

The limitation of existing experiments with IACTs - HESS [1], MAGIC [2] and VERITAS [3] -is their insufficient sensitivity to gamma radiation with an energy $\mathrm{E}>15-20 \mathrm{TeV}$ due to the small size of the detector area $\sim 0.05 \mathrm{~km}^{2}$. This makes it impossible to detect small flux of the gamma radiation at $\sim 100 \mathrm{TeV}$.

For this reason the TAIGA (Tunka Advanced Instrument for Gamma Astronomy) setup includes a wide-angle Cherenkov detector HiSCORE timing array with an energy threshold for EAS gamma rays E 30 $\mathrm{TeV}$ and for the hadron CR component E 100 TeV. The HiSCORE has a viewing angle is about $0.6 \mathrm{sr}$ and an angular resolution of $0.1-0.2^{\circ}$ as well as the IACTs. The IACTs will increase the sensitivity of the array in the low-energy region, while allowing to retain a high angular resolution [4]. This hybrid method allows to reduce the background of hadronic CR showers by factor $\sim 100$ at the energy of $100 \mathrm{TeV}$ for point-like sources and to increase the distance between IACTs up to $600 \mathrm{~m}$ without loss of the EAS accuracy reconstruction in the measurements of spatial and energy characteristics. For the more distant TAIGA future it is expected to increase the experimental area up to $5 \mathrm{~km}^{2}$ with the detection sensitivity of such array for local sources in the energy range 30-200 TeV will be $\sim 10^{-13} \mathrm{erg} \cdot \mathrm{cm}^{-2} \cdot \mathrm{s}^{-1}$ for 500 hours of observation or 10 detected events [5].

\section{The Imaging Atmospheric Cherenkov Telescope}

The IACT was fabricated mainly at JINR on the basis of the HEGRA [6] telescope design. The IACTs are of Davis-Cotton type with alt-azimuth mount, total mirror area of $9.6 \mathrm{~m}^{2}$ (34 mirrors, each has diameter $0.60 \mathrm{~m}$ ), the driving angular accuracy is up to $0.02^{\circ}$. The imaging camera with weight $\sim 200 \mathrm{~kg}$ and diameter of sensitive area of $\sim 0.8 \mathrm{~m}$ [7] is based on a matrix of PMTs with FrontEnd and DAQ electronics and is fixed at the focal length of $4.75 \mathrm{~m}$ in front of the mirror dish. The EAS Cherenkov light is focused on the camera by the 34 mirror facets of $9.5 \mathrm{~m}$ curvature radius. The mirrors for the first IACT were obtained from "Galactica” (Armenia), mirrors for the second IACT are from "Media Lario Industries” (Italy).

The imaging camera comprises 560 PMTs of type XP1911. Each PMT has 15 mm photo-cathode diameter and is equipped with a Winston cone with a diameter of $30 \mathrm{~mm}$ to enhance the sensitive area by factor of 4 , designed in such a way that a single pixel of the camera views the whole reflector of the telescope. An an gular size view of a single pixel is $0.36^{\circ}$. The total FOV of the IACT is of $\sim 9.5^{\circ}$ [8].
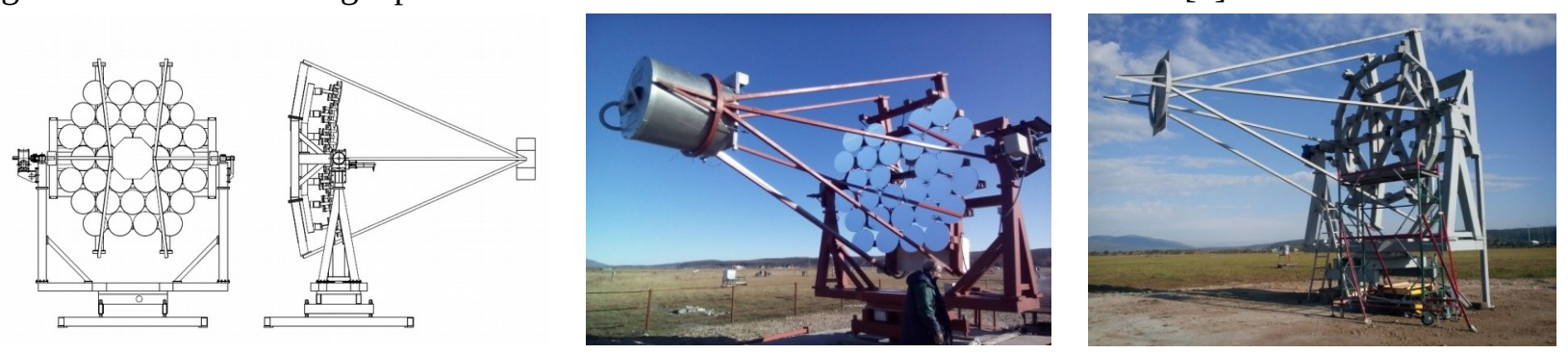

Figure 1: The TAIGA IACT: technical drawing (left), the first IACT (middle) and the second IACT structure (right) in the Tunka valley.

\section{The focusing mirror production technology}

A technology for the production of focusing glass mirrors and bending was developed at JINR. We briefly describe production and test procedure. 
Initially, the steel billet is subjected to controlled heating in the furnace (Figure 2 (left)) for 48 hours. The glass disk deforms and takes the spherical shape of steel mold under the action of gravity. A special mode of temperature changing makes it possible to achieve a sufficiently smooth surface and to avoid gluing of the billet to the mold.

Current tests are aimed to increase the smoothness of the final product and to avoid the subsequent glass polishing and to minimize the curvature errors to the limits acceptable for the TAIGA-IACT. The diameter of the focal spot should not be much larger than 3-6 mm. The initial glass disk's quality with a diameter of $600 \mathrm{~mm}$ thickness uniformity of about $0.02 \mathrm{~mm}$ allows to expect that the bending procedure will minimize subsequent processing. The polishing machine with the aluminum alloy polishing disk is presented in Figure 2 (right).
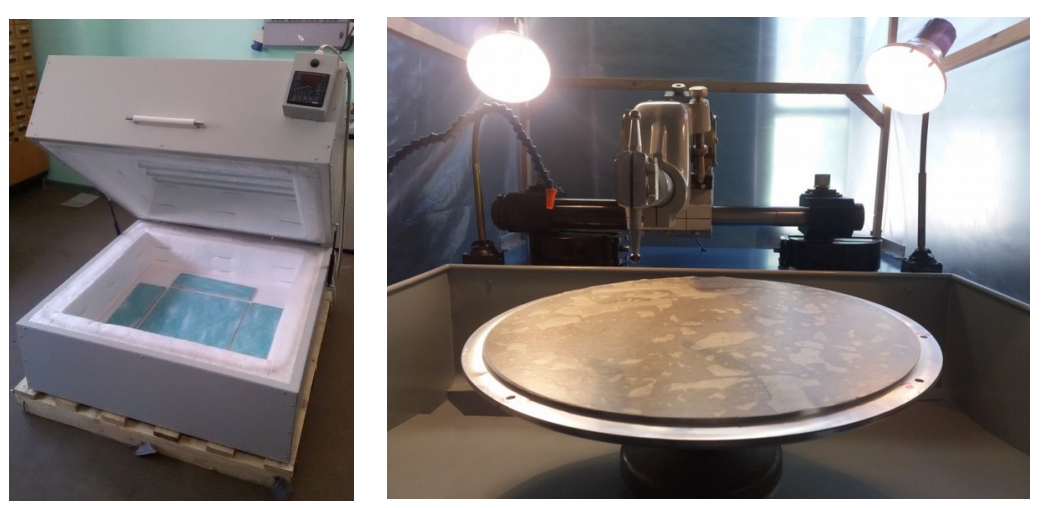

Figure 2: Glass bending oven (left), polishing machine (right)

Below, we describe some first measurements made with the JINR mirror prototype and the composite mirrors of the Media Lario Industries (Italy). To check the surface shape quality, a special stand was build, as shown in Figure 3. The stand allows to obtain series of images and then to process them to analyze the intensity distribution. The spot shape and relative intensity distributions of the JINR glass mirrors was compared with the spot shape and relative intensity distributions of the composite mirrors from Media Lario Industries.

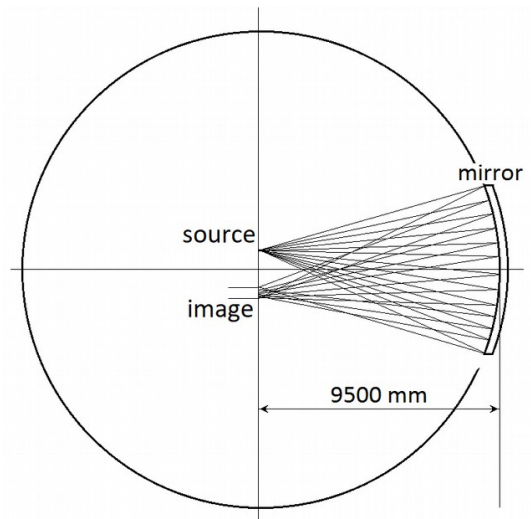

Figure 3: Scheme of the stand for the surface quality check. The photo camera with the opened matrix is near the focal point 
In Figure 4 the results of the mirror measurements are presented. The spot shape and the relative intensity distributions were measured with a NICON D70 camera without objective unit and then processed.
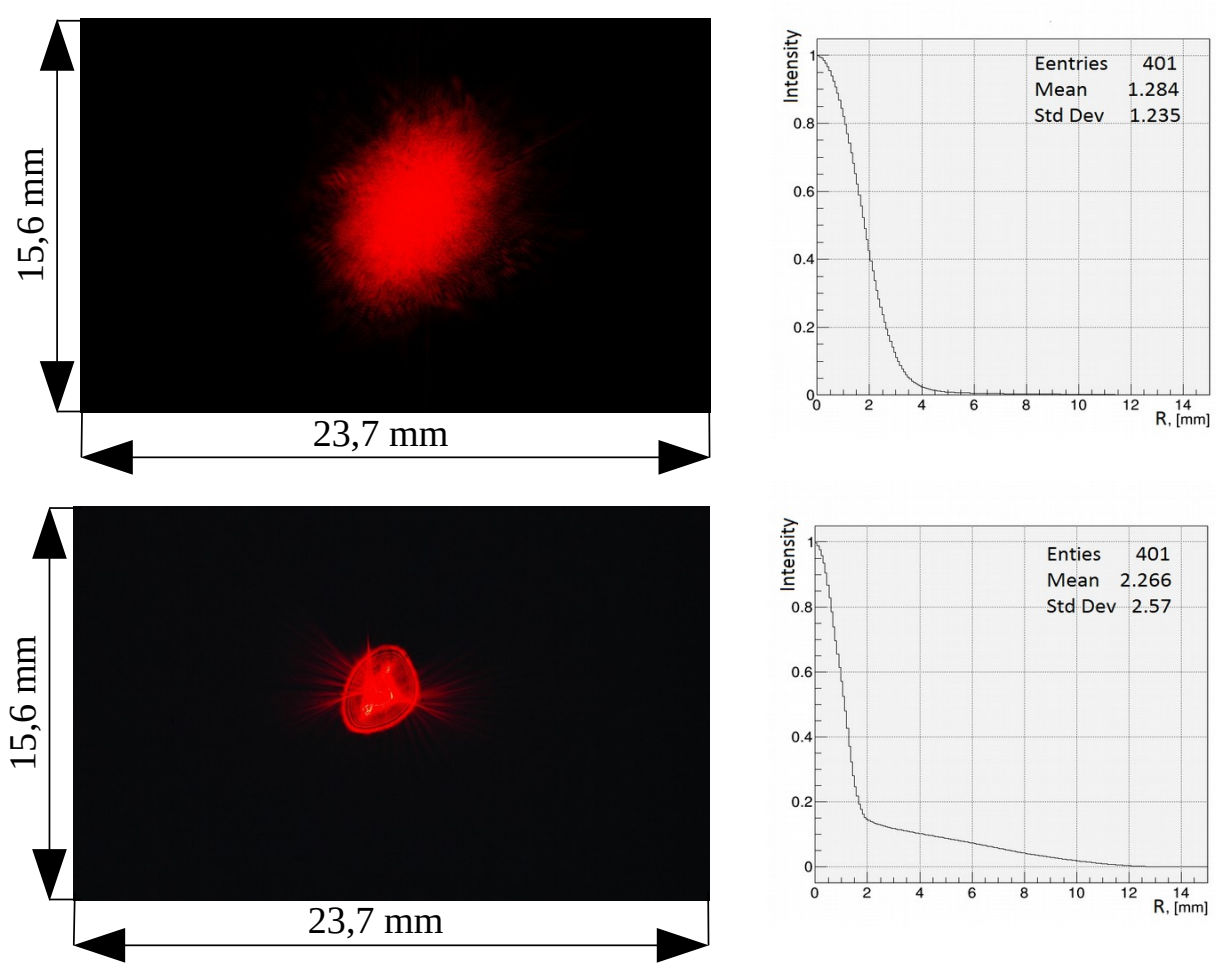

Figure 4: The spot shape and the relative intensity distribution measurements for the composite mirror of the Media Lario company (top) and the prototype mirror fabricated at JINR (bottom). The real length and width of the images is 23,7 and $15,6 \mathrm{~mm}$, respectively.

Spot sizes of both mirrors have comparable values and correspond well to the pixel size of the IACT camera. The main difference is cost of the mirror production that will be much cheaper for mirrors from JINR.

\section{IACT pointing performance}

The telescope tracking system consists of two Phytron hybrid stepper motors and two 17-bit shaft encoder and limit switches connected to the PhyMOTION control unit [9]. A CCD-camera Prosilica GC1380 is installed on the telescope dish at a distance of $1 \mathrm{~m}$ from the telescope optical axis. The CCD-camera is used to determine the telescope pointing direction by its images and to perform calibration measurements. The trajectory for the telescope movement is calculated using the SOFA software and applying a pointing model. The telescope tracking system is controlled by software developed within the EPICS framework [10]. As a test of the telescope pointing, special tracking runs are performed. These runs include the cross-like scan (see Figure 5, left) of a bright star near the central PMT of the camera. During the scan, the anode current of the central PMT as well as the driving system information, including encoder data and CCDcamera images, are recorded. For each measurement, the bright star position on the focal plane is calculated to obtain the current profiles along the $\mathrm{X}$ and $\mathrm{Y}$ axis in the PMT coordinate system. From the obtained current profiles along the $\mathrm{X}$ and $\mathrm{Y}$ axis, the center of gravity is calculated, see Figure 5 (right). The difference between the PMT position and the center of gravity is the telescope mispointing value. This calibration procedure is performed several times at the beginning and the end of each 2-3 weeks observation period.

During the central pixel-scans described above, also other stars and their corresponding current profiles 
are obtained. Using the method described for the central pixel, the differences between expected and measured center of gravity are obtained. The distribution of points measured between November 2018 and March 2019 is shown in Figure 6. It includes measurements with current profile amplitudes larger than 0.3 $\mu \mathrm{A}$ and a scan track distance to the pixel center of less than $0.1^{\circ}$.

A main purpose of the TAIGA observatory is the investigation of coincident events [4,5,18], i.e. events that are simultaneously detected with both the TAIGA-HiSCORE Cherenkov array and the TAIGA-IACTs. Figure 7 demonstrates this approach, by plotting on the RA-DEC (right ascension, declination) sky the arrival directions of cosmic ray showers that are detected and fully reconstructed with HiSCORE. Data for 14 TAIGA runs, recorded in the 2017/18 season, and with the IACT operating in precision Crab-nebula tracking mode are shown, selecting HiSCORE events with an IACT trigger within an $1.9 \mu$ sec time window around the HiSCORE trigger (coincident events). The dashed circle gives the $4.8^{\circ} \mathrm{FoV}$-radius of the IACT.
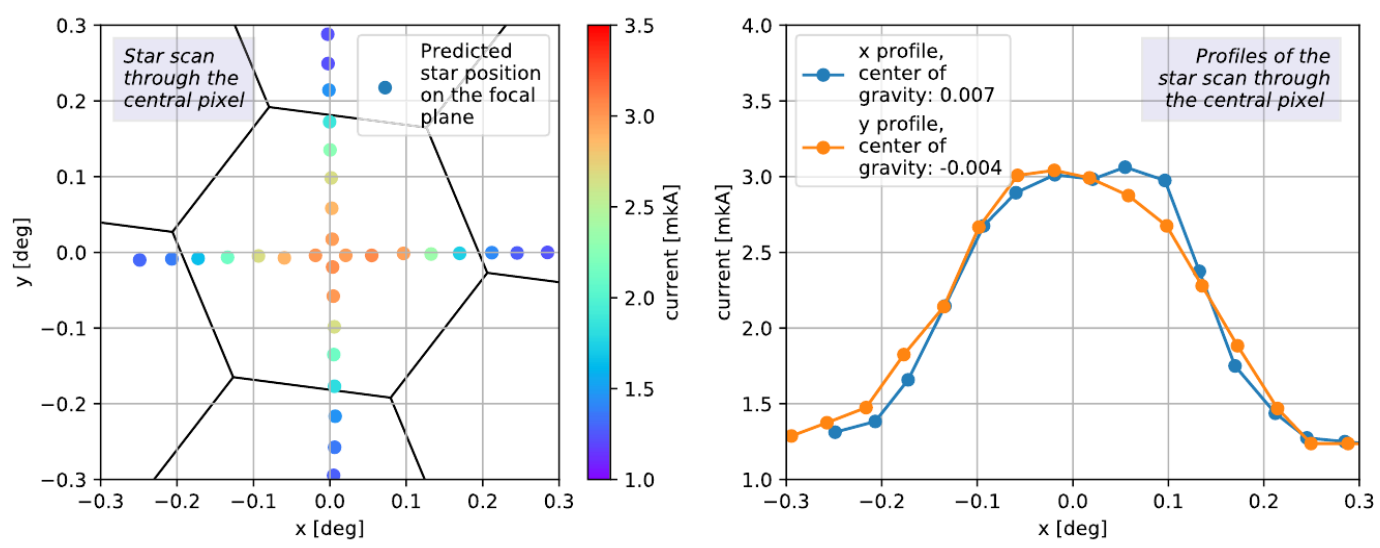

Figure 5: Telescope pointing test based on the measurement of anode current during special tracking runs. Left: calculated positions of a bright star on the focal plane are shown by dots. Current values on the central PMT are given by the color scale. Black lines indicate the camera pixels. Right: current profile as measured on the central PMT for a specific along $\mathrm{x}$ - and $\mathrm{y}$-direction.

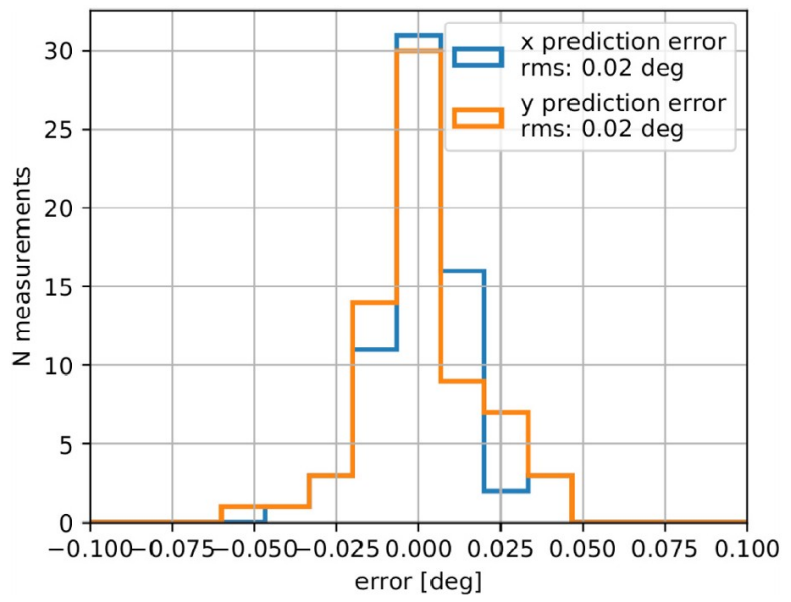

Figure 6: Distribution of differences between center of gravity, calculated from measured current profiles and pixel positions for central and non-central pixels. The distribution includes measurements at telescope inclinations between 40 and $80^{\circ}$, for November 2018 to March 2019. 


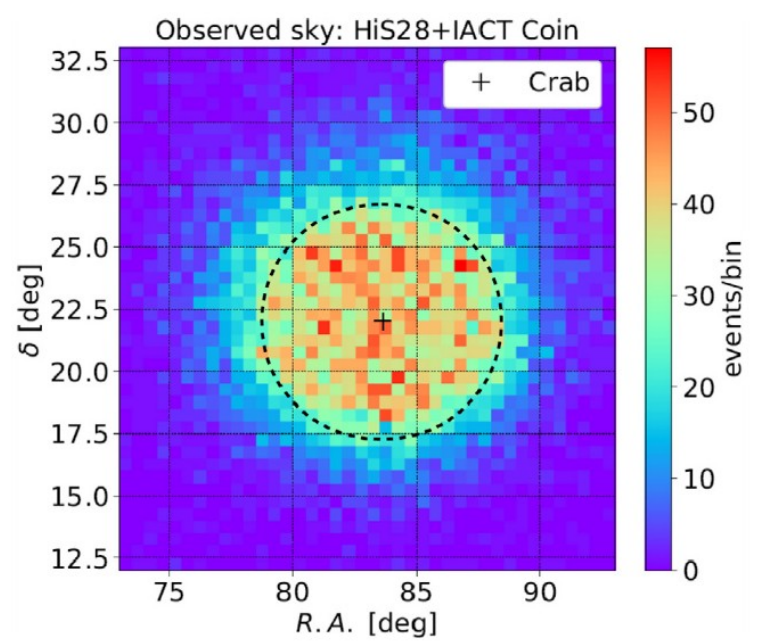

Figure 7: Coincident events sky map. Events triggered by both IACT and TAIGA-HiSCORE array. The arrival direction is determined with HiSCORE. Events are concentrated within the acceptance of the telescope, indicated by the dashed circle of $4.8^{\circ}$ radius.

\section{Conclusions}

The TAIGA observatory combines imaging and non-imaging technology to detect gamma rays at energies above $10 \mathrm{TeV}$ [18]. The first of three planned TAIGA IACT has been operating since 2017 in the Tunka valley. A second IACT structure is ready for camera installation at Tunka, the third telescope structure will be mounted in fall 2019. The telescope pointing precision has been studied over two seasons, and fulfills TAIGA-requirements. The collaboration has developed a technology for IACT glass mirror facets manufacturing and quality control.

We are considering to test alternative camera technologies (e.g. as developed for SST-1M/CTA [19]) at the TAIGA site. After adapting this camera for the local environmental conditions, this would allow for cross-calibration, and for recording the full waveform for each camera pixel. MC-simulation of resulting sensitivity improvements are in progress.

\section{Acknowledgements}

This work is supported by the Russian Science Foundation (grant 19-72-20067 (Section 4), agreement 19-72-20173), the Russian Foundation for Basic research (grant 18-32-00460), the Russian Federation Ministry of Education and Science (agreement 3.9678.2017/8.9, 3.904.2017/4.6, 3.6787.2017/7.8, 3.6790.2017/7.8, 3.5917.2017/Bch) and by the European Union's Horizon 2020 program (grant 653477).

\section{References}

[1] A. Förster et al., Gamma-ray astronomy with H.E.S.S., Nuclear Instruments and Methods in Physics Research A (2014), Volume 766, 1 December 2014, Pages 69-72.

[2] Javier Rico, on behalf of the MAGIC Collaboration, Overview of MAGIC results, Nuclear and Particle Physics Proceedings, Volumes 273-275, April-June 2016, Pages 328-333.

[3] Jean-Francois Rajotte for the VERITAS Collaboration, Upgrade and performance of the VERITAS telescope array, Nuclear Instruments and Methods in Physics Research, A (2014), Volume 766, 1 December 2014, Pages 6164.

[4] N. Budnev et al. (TAIGA Coll.), The TAIGA experiment: from cosmic ray to gamma-ray astronomy in the Tunka valley, Journal of Physics: Conference Series 718 (2016) 052006. 
[5] E. Postnikov et al. (TAIGA Coll.), Commissioning the joint operation of the wide angle timing HiSCORE Cherenkov array with the first IACT of the TAIGA experiment, PoS(ICRC2017)756.

[6] R. Mirzoyan et al., The First telescope of the HEGRA air Cherenkov imaging telescope array, Nuclear Instr. And methods A351 (1994) 513-526. DOI: 10.1016/0168-9002(94)91381-1.

[7] I.I. Yashin et al. (TAIGA Coll.), The TAIGA project, Journal of Physics: Conference Series 675 (2016) 032037.

[8] I.I. Yashin et al. (TAIGA Coll.), Imaging camera and hardware of Tunka-IACT, PoS(ICRC2015)986.

[9] N. Budnev et al. (TAIGA Coll.), TAIGA - A hybrid array for high-energy gamma astronomy and cosmic-ray physics, Nuclear Instr. and Methods in Physics Research, A (2019), https://doi.org/10.1016/j.nima.2019.04.067 (Article in press).

[10] D. Zhurov et al. (TAIGA Coll.), Phys. Conf. Ser. 1181 (2019) 012045.

[11] T.C. Weekes et al., ApJ, 342379 (1989).

[12] I Astapov et al. (TAIGA Coll.), Bull. Russian Academy of Sciences: Physics 81460 (2017).

[13] F. Aharonian, Very High Energy Cosmic Gamma Radiation. A Crucial Window on the Extreme Universe, World Scienti_c, River Edge, 2004.

[14] D. Heck et al., Tech. Rep. FZKA-6019, Forschungszentrum Karlsruhe (1998).

[15] S. Blin, P. Barrillon and C. de la Taille, JINST 5 C12007 (2010).

[16] N. Lubsandorzhiev et al. (TAIGA Coll.), PoS 301 PoS(ICRC2017)757 (2017).

[17] R. Mirzoyan et al., NIMA 387 74-78 (1997).

[18] N. Lubsandorzhiev et al. (TAIGA Coll.), The hybrid installation TAIGA: design, status and preliminary results, PoS(ICRC2019)729, these proceedings.

[19] M. Heller et al. An innovative silicon photomultiplier digitizing camera for gamma-ray astronomy, Eur. Phys. J. C77 (2017) 1, 47, https://arxiv.org/pdf/1607.03412.pdf 